\title{
Robotic surgery for locally advanced non-small cell lung cancer
}

\author{
Piergiorgio Muriana $^{1} \wedge$, Gianluca Perroni ${ }^{2}$, Pierluigi Novellis ${ }^{1}$, Giulia Veronesi ${ }^{1,3}$ \\ ${ }^{1}$ Department of Thoracic Surgery, IRCCS San Raffaele Scientific Institute, Milan, Italy; ${ }^{2}$ Department of Thoracic Surgery, Humanitas Clinical and \\ Research Center, Milan, Italy; ${ }^{3}$ Vita-Salute San Raffaele University, Milan, Italy \\ Contributions: (I) Conception and design: G Veronesi, P Muriana; (II) Administrative support: G Veronesi; (III) Provision of study materials or \\ patients: G Veronesi, G Perroni, PN; (IV) Collection and assembly of data: P Muriana; (V) Data analysis and interpretation: P Muriana, G Veronesi; \\ (VI) Manuscript writing: All authors; (VII) Final approval of manuscript: All authors. \\ Correspondence to: Piergiorgio Muriana, MD. Department of Thoracic Surgery, IRCCS San Raffaele Scientific Institute, Via Olgettina 60, 20132 \\ Milan, Italy. Email: muriana.piergiorgio@hsr.it.
}

\begin{abstract}
Stage III non-small cell lung cancer (NSCLC) is a heterogeneous group of diseases including large masses, mediastinal lymph node involvement, and invasion of major mediastinal structures. Robotic surgery is a minimally invasive approach recognized as an equivalent alternative to open surgery in case of early stage NSCLC. An increasing number of studies also assert that robotics is a reasonable option for the treatment of locally advanced disease in the setting of multimodality therapy. Major advantages of the technique are increased radicality compared to video-assisted thoracic surgery (VATS), fast postoperative recovery and better compliance to adjuvant therapies compared to open. In this paper we present some technical aspects of the robotic lobectomy in locally advanced disease, we discuss different robotic approaches and review the literature of robotic treatment of lung cancer patients. To describe the technique, we use two case studies of patients affected by locally advanced NSCLC operated with robotic surgery. A patient with right upper lobe (RUL) pulmonary adenocarcinoma and single N2-station metastasis underwent robotic lobectomy following response to induction chemo-radiotherapy. A radical excision of the tumor was obtained with clear margins and no evidence of residual disease at pathologic examination. In the second case, a diagnosis of N2 lymph node metastasis (station R4) from pulmonary adenocarcinoma was made in a patient with primary occult tumor. After induction treatment, the patient underwent robotic radical mediastinal lymph node dissection with curative intent. Unexpected partial invasion of the wall of superior vena cava (SVC) was safely managed with tangential resection. Despite the problems of costs, availability, and learning curve, the Authors believe that robotic surgery has indisputable advantages to obtain a radical resection in case of locally advanced NSCLC.
\end{abstract}

Keywords: Lung cancer; locally advanced; minimally invasive surgery; robotic surgery; multimodality treatment

Received: 27 April 2020; Accepted: 26 May 2020; Published: 20 July 2021.

doi: $10.21037 /$ jovs-20-114

View this article at: http://dx.doi.org/10.21037/jovs-20-114

\section{Introduction}

About $27 \%$ of the patients affected by non-small cell lung cancer (NSCLC) have locally advanced disease at the time of diagnosis (1). Although all these patients are classified as stage III of the Tumor-Node-Metastasis (TNM) staging system (2), this is a rather heterogeneous group of diseases comprising large tumors, neoplasms invading the mediastinum and the chest wall, and patients with mediastinal lymph node metastasis associated with small or even undetectable primary lesion (3).

Given the variety of the clinical presentation, it is nowadays widely accepted that stage III NSCLC requires

^ ORCID: 0000-0003-1341-1071 
a multimodal therapeutic approach in the setting of a multidisciplinary team with thoracic surgery, medical oncology and radiotherapy specialists (3). A combination of local and systemic treatments is needed to achieve a radical control of the disease and to reduce the risk of loco-regional and distant recurrence.

NCCN (4) and ESMO guidelines (5) recommend induction chemotherapy with platinum-based regimens with or without concurrent radiation therapy prior to surgical exploration in case of resectable T3 and T4 tumors, or N2-station involvement. In addition, in the last few years some trials have been investigating the role of anti-PD-L1 checkpoint inhibitors as neoadjuvant treatment (5). Adjuvant therapies are usually suggested to consolidate the results of local treatment (6). In selected patients with good performance status and resectable disease, surgery has been even evaluated as the initial treatment after careful multidisciplinary team discussion, e.g. in case of single N2-station involvement of upper lobe tumors (7), but the literature is still lacking prospective trials confirming the validity of such approach compared to traditional schemes (8).

Surgery of locally advanced disease has been traditionally considered challenging. The reasons for this are manifold. The surgeon has to face with bulky and necrotic lesions, sometimes superinfected, that frequently invade major mediastinal structures. Moreover, induction therapy may induce high-grade fibrosis that hinder a safe isolation of hilar structures $(8,9)$. High morbidity and mortality rates are expected, in particular in those patients requiring extended resections (10). It is a common opinion that, when a pneumonectomy is required to achieve radicality, surgery should be contraindicated. However, some Authors reported acceptable rate of postoperative complications and mortality following an accurate selection of patients undergoing major pulmonary resection after neoadjuvant therapy $(3,11)$.

Hence, in patients with good performance status, oligometastatic mediastinal nodal involvement, and a good response to induction therapy, it is reasonable to consider surgery with appropriate timing arranged by the multidisciplinary board as a therapeutic option (8). In this context, video-assisted thoracic surgery (VATS) emerged as a potential alternative equivalent to thoracotomy in terms of long-term survival in selected favorable cases (12). Apart from the well-known effects on perioperative outcomes (lower incidence of postoperative complications, faster recovery, improvement of the quality of life) (13), another advantage of VATS is a reduction of surgical trauma and a lowering of the inflammatory status (14). In fact, it was demonstrated that increased systemic inflammation is predictive of reduced survival in surgically treated NSCLC patients (15). Drawbacks of VATS approach are the long learning curve to reach the skills required to afford complex surgical procedures and the technical limitations related to the rigid instruments and suboptimal 2-dimensional view.

Since its introduction in the clinical practice, roboticassisted thoracic surgery (RATS) showed several advantages, including 3-dimensional magnified view, better ergonomics, natural and wide range hand movements, and tremor filtration $(16,17)$. Compared to open surgery, patients who undergo RATS lobectomy for lung cancer have lower postoperative complications and perioperative mortality (18), a comparable number of lymph nodes removed, and shorter hospital stay (16). RATS has been shown to be superior to VATS in the terms of number of lymph node stations harvested during lobectomy for early-stage disease (19). Moreover, the learning curve of RATS lobectomy seems to be shorter than that of VATS lobectomy (20).

It has been generally accepted that the main indication for robotic-assisted pulmonary resection is the treatment of small stage I and II tumors with minimal burden of hilar nodal disease. However, several Authors assert that RATS, due to its advantages as the more accurate lymph node dissection, should really be applied to locally advanced disease, therefore extending the indication to robotic approach beyond early-stage NSCLC (17,21-25).

In this paper, we report a review of the literature on the role of RATS in locally advanced cases and describe the technique of two case studies of patients affected by locally advanced NSCLC operated with robotic surgery in the setting of multimodality treatment.

\section{Robotic surgery for locally advanced NSCLC: literature review}

According to data from STS database (26), in 2013 about $14 \%$ of all minimally invasive pulmonary lobar resections for early-stage NSCLC were carried out using robotics.

Nonetheless, indication for robotic approach progressively broadened to more advanced disease requiring complex procedures, such as surgery for Pancoast tumor, bronchoplastic procedures and double-sleeve lobectomies (27-32).

A considerable proportion of locally advanced NSCLC cases is represented by patients with N2-disease. As previously shown, patients with bulky involvement of the 


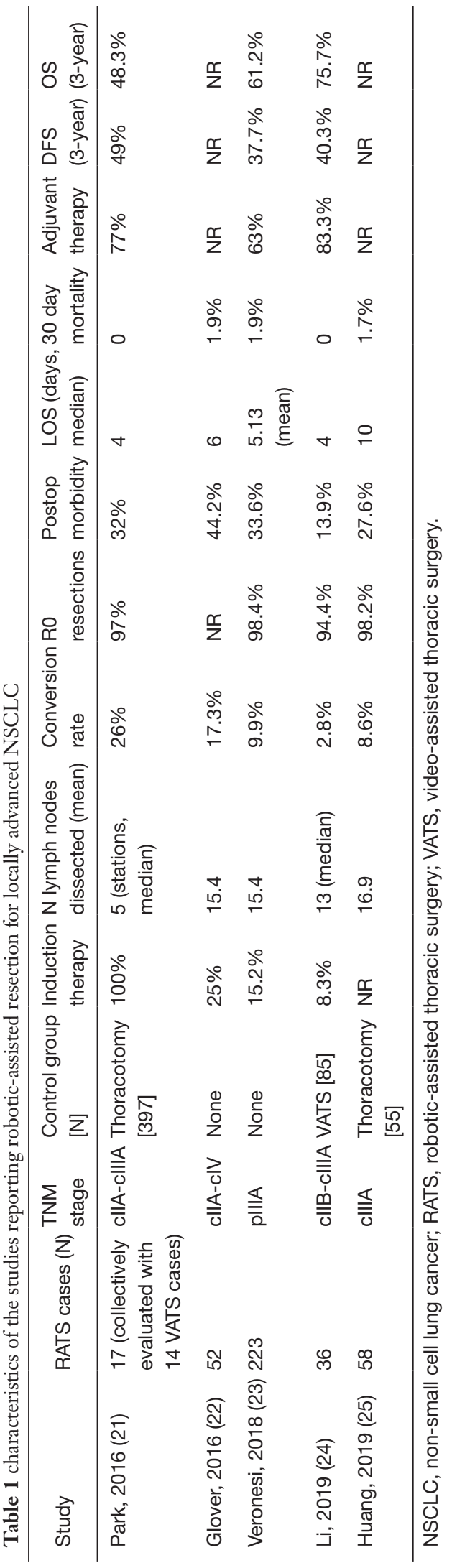

mediastinum or multi-station nodal disease at staging should preferably undergo a definitive multimodal nonsurgical treatment because of poor prognosis in case of resection (8).

An analysis conducted on over 3,000 VATS lobectomy cases enrolled in the Italian National Registry (Italian VATS-Group) reported, however, that more than $6 \%$ of patients had nodal upstaging for unexpected $\mathrm{N} 2$ positivity at final histology (33). Nonetheless, the latest revision of $\mathrm{N}$ descriptor of TNM staging of lung cancer carried out by the International Association for the Study of Lung Cancer (IASLC) (34) gave evidence of significant survival difference depending on the number of metastatic lymph node stations involved by the disease. In particular, patients with single N2-disease without associated positive N1-stations (N2a1) showed survival rates that were anyway comparable to those with multiple level N1-disease.

On this basis, there is common consensus that patients with limited N2-disease ought to be selected for a multimodal treatment with induction therapy followed by surgery (35). Overall 5 -year survival in patients with partial response to induction treatment (i.e., persistence of N2disease) is about $30 \%$ (8), but it can increase up to $50 \%$ in case of complete pathologic response (ypT0, ypN0) $(36,37)$.

The number of single and multicentric studies of robotic surgery for locally advanced lung cancer is still limited but it increased in the last years (Table 1). In 2016, Park and colleagues first reported their experience with 17 patients previously treated with neoadjuvant therapy who underwent robotic lobectomy for stage II and IIIA NSCLC (21). Data of robotic procedures were collectively analyzed with those of additional 14 patients treated by means of VATS, and compared to a group of 397 patients who underwent open thoracotomy. No difference was found in oncological outcomes (complete resections, disease-free and overall survivals), as well as in the rate of postoperative complications. The minimally invasive group, however, showed a shorter hospital stay $(\mathrm{P}<0.001)$. Although there was no difference in the distribution of pathologic stage between the groups, $77 \%$ of patients in the minimally invasive group were able to receive adjuvant therapy $v s$. $38 \%$ in the open approach group $(\mathrm{P}<0.001)$, probably because of the reduced surgical trauma.

In a single-center retrospective analysis conducted by Glover et al. on 52 patients with locally advanced NSCLC resected with robotic surgery (22), the number of recurrent laryngeal nerve or airway injuries was significantly higher among the patients who received induction therapy. 

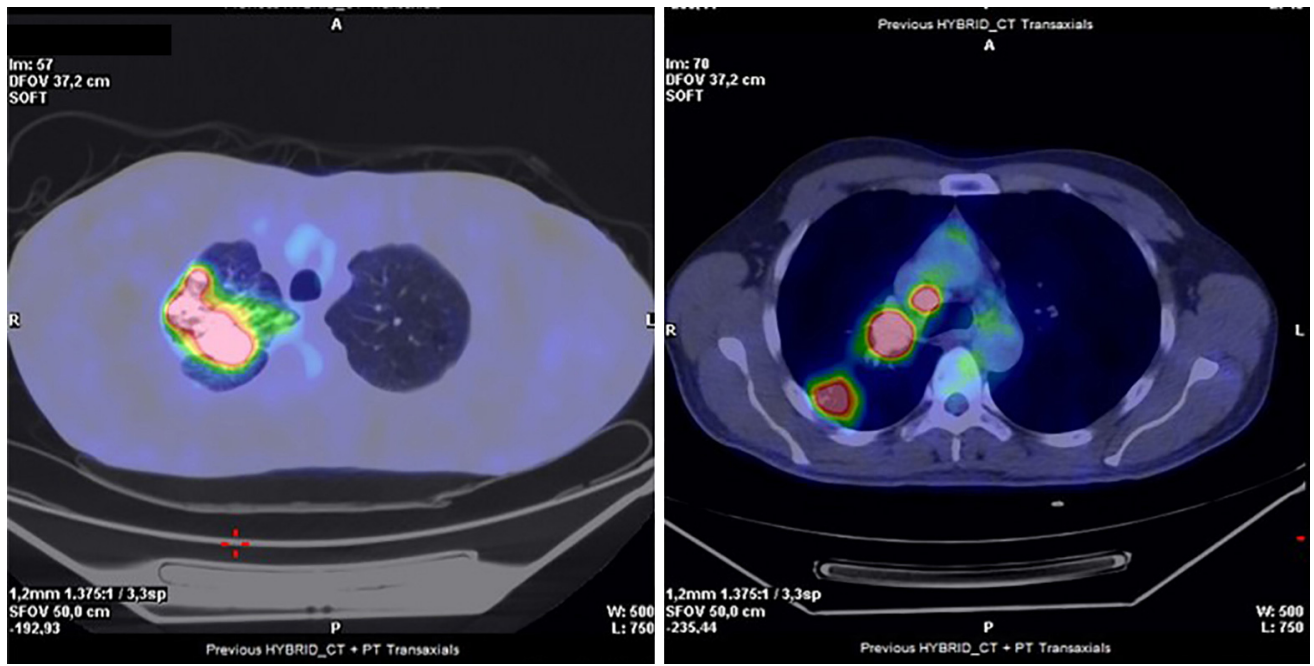

Figure 1 Case 1, PET-CT scan showing a RUL mass with enlarged hilar and mediastinal lymph node metastasis. RUL, right upper lobe.

However, the Authors pointed out that, regardless of the neoadjuvant therapy status, the number of resected lymph nodes was comparable between the groups.

In 2018 our group reported the results of a multicentric retrospective analysis of the safety of robotic surgery in stage III NSCLC (23). The study enrolled 223 patients with clinically evident or occult N2-disease; 34 out of 72 patients with N2 involvement underwent neoadjuvant treatments. Perioperative complications and survival were consistent with open surgery data reported in the literature. The need of conversion ( $10 \%$ overall), perioperative complications, and $\mathrm{R} 0$ resection rates were similar regardless of neoadjuvant therapy status. Sixty-three percent of the patients received postoperative adjuvant therapy.

In the study of $\mathrm{Li}$ et al. (24), 36 patients with locally advanced NSCLC and nodal involvement resected by means of RATS at Shanghai Chest Hospital over a 3-year period had a higher number of lymph nodes harvested and a shorter postoperative in-hospital stay than a comparable group of patients treated with videothoracoscopy. Conversion to thoracotomy was necessary only in $2.8 \%$ of patients, and in no case because of bleeding. Of note, only 3 patients in the RATS group and one patient in the VATS group had previously been treated with neoadjuvant therapies, due to the high prevalence of N1-disease in the cohort, and the institutional choice of upfront surgery in patients with small N2 lymph nodes. Overall survival 3 years after surgery exceeded $75 \%$ in both groups.

Recently, preliminary data of a randomized controlled trial comparing RATS and open surgery for the treatment of clinical stage IIIA-N2 NSCLC have been released (25). Fifty-eight patients received robotic-assisted lobectomy and systematic lymph node dissection. Total number of lymph nodes and number of lymph node stations dissected was similar in both groups, but RATS resulted in significantly less intraoperative blood loss, chest tubing, and better postoperative pain control.

\section{Technical aspects and clinical cases}

\section{Case 1}

\section{Case presentation}

A 49-year-old male patient, active smoker, was admitted to our Department for a suspected right upper lobe (RUL) lung cancer found after the onset of hemoptysis. His medical history was silent. Contrast-enhanced chest CT scan showed a $5.5 \mathrm{~cm}$ diameter solid lesion with irregular borders in continuity with enlarged hilar and mediastinal lymph nodes. Abdominal sections did not show lesions suspected for metastasis. Intense radiotracer uptake of the pulmonary mass and of lymph node stations R10 and R4 was evident at whole-body 18-FDG-PET scan (Figure 1). In consideration of the clinical staging (cT3, cN2), brain imaging with MRI was indicated and resulted negative for metastasis.

The patient underwent bronchoscopy with endobronchialultrasound transbronchial needle aspiration (EBUSTBNA). Bronchial biopsy of thickened tissue at the origin of the RUL bronchus was positive for adenocarcinoma G3 

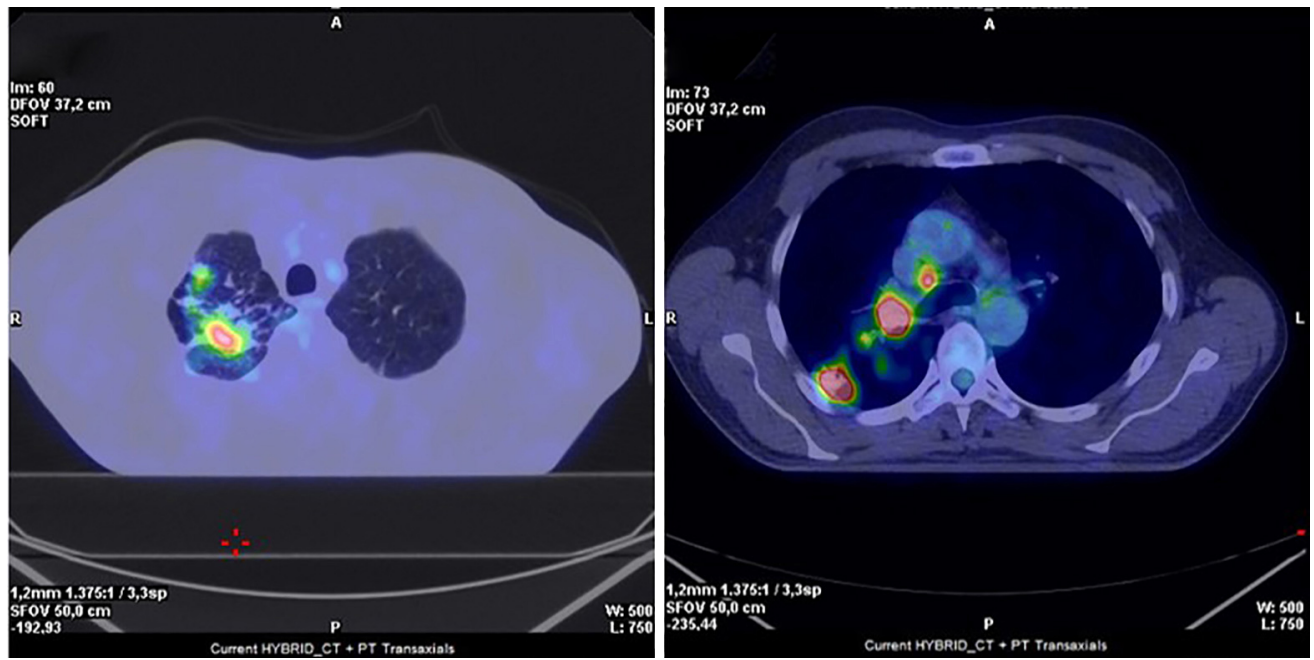

Figure 2 Case 1, following a bronchoscopic diagnosis of cT3, cN2 pulmonary adenocarcinoma, the patient was submitted to induction chemo-radiotherapy. Restaging PET-CT scan showed a good response to the treatment with downstaging of the disease.

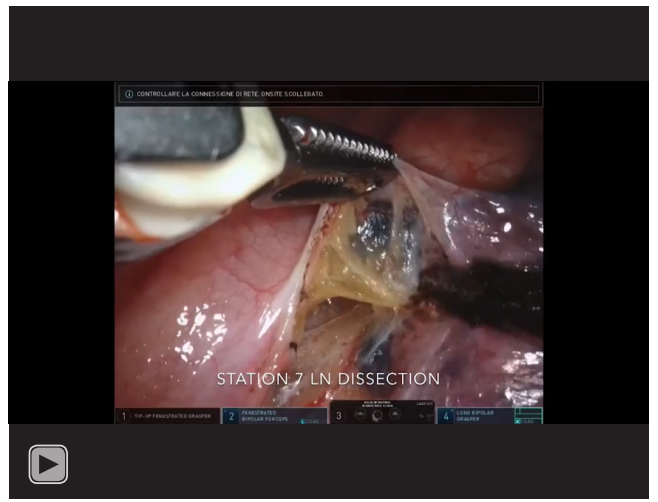

Video 1 Case 1, robotic-assisted right upper lobectomy after neoadjuvant chemo-radiotherapy.

(TTF-1+, p63-, synaptophysin-). Cytological and histologic specimens of R4 and 7 lymphatic stations resulted however inadequate. The patient was then scheduled for a cervical mediastinoscopy, but he refused to undergo the procedure.

Following multidisciplinary counselling, the patient was offered an induction treatment with cisplatin-vinorelbine combination and concurrent radiotherapy. After 2 cycles of chemotherapy and 50 Gy radiation, restaging with chest CT scan, PET scan, and bronchoscopy showed a good morphologic, metabolic (Figure 2), and endoscopic response to the treatment. Considering the downstaging of the disease (ycT2b, ycN1/2 for a single station R4) and the good performance status of the patient, indication to radical surgical treatment with robotic-assisted RUL lobectomy was given.

\section{Preoperative work-up and anesthesia management}

Routine exams include chest X-ray, EKG, complete blood tests, and baseline pulmonary function tests (spirometry + DLCO assessment). Additional cardiac and respiratory investigations are performed in selected patients following ACCP guidelines for preoperative evaluation of candidates to pulmonary resection (38).

In all patients, general anesthesia is administered and a Robertshaw tracheal double-lumen tube is placed and controlled. After positioning the patient in lateral decubitus, intercostal block from $\mathrm{T} 3$ to $\mathrm{T} 8$ with ropivacaine is delivered by the anesthetist before incision.

\section{Surgical technique (Video 1)}

RATS surgery is performed with Da Vinci Xi robotic system (Intuitive Surgical Inc., Sunnyvale, CA, USA). We use the Park-Veronesi four-arm robotic technique (16), making an anterior utility incision in the fourth intercostal space with soft tissue retractor placement and 3 additional $8 \mathrm{~mm}$ incisions at the level of the $7^{\text {th }}-8^{\text {th }}$ intercostal space around the tip of the scapula for robotic arm ports, $30^{\circ}$ camera, and for the introduction of thoracoscopic instruments by the table assistant (Figure 3). Dissection of hilar structures and manipulation of the pulmonary parenchyma is provided with a curved bipolar dissector, a fenestrated bipolar grasper, and a Tip-Up fenestrated grasper. 


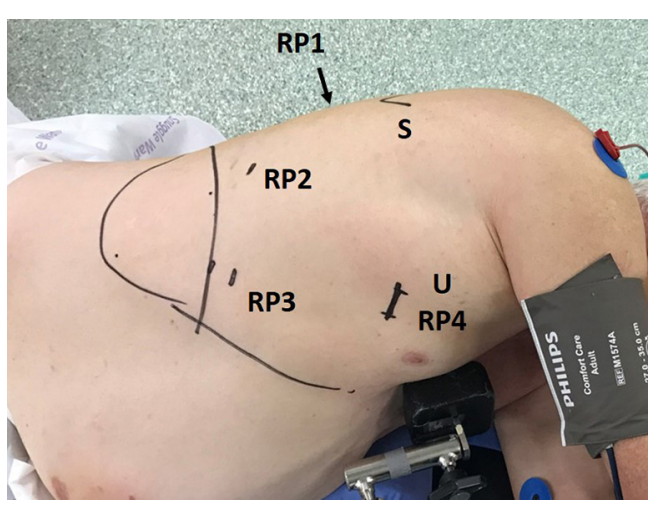

Figure 3 Patient position and surgical accesses (U: utility incision, RP 1-2-3-4: robotic arm ports, S: scapula).

In the present case, preliminary thoracoscopic inspection ruled out the presence of pleural metastases. Pulmonary parenchyma was mobilized from parietal adhesions.

Usually, in case of early-stage disease, the operation starts with the isolation of hilar vascular structures with an anterior-to-posterior fissureless approach (39). Following induction treatment, a pathologic assessment of the response to chemo-radiotherapy is crucial to confirm the indication to lobectomy. Hence, in this case, a radical nodal dissection was first of all performed. Mediastinal pleura was opened and station 7, R4, and R2 lymph nodes were harvested along with surrounding fat. The curved bipolar dissector enables a safe dissection even in presence of fragile tissue secondary to neoadjuvant therapy. To obtain proper exposure of Barety lodge, azygos vein was resected with an Endo GIA stapler (Tri-Staple ${ }^{\mathrm{TM}}$ Technology, Medtronic). Hemostatic sponge was placed in empty spaces left from the specimen in order to control local bleeding. The fissure between the upper lobe and the inferior lobe was dissected and station R11 nodes were removed. Frozen-section of all the nodes resulted free from metastatic invasion.

After the identification of the S6 segmental artery, the posterior portion of the fissure was divided with staplers (purple cartridge). A small ascending RUL artery was isolated and sectioned after ligation. Hem-o-Lok robotic clips (Teleflex Inc., Wayne, PA, USA) or staplers might be alternatively used in case of larger vessels.

The lung was afterwards retracted posteriorly so as to expose the anterior aspect of the hilum. The mediastinal pleura was incised laterally to the phrenic nerve. The RUL pulmonary vein was dissected and the middle lobe branch was identified and spared. The bipolar grasper was passed underneath the vein, encircling the vessel with a silicone loop. Traction was applied to the loop to ease the passage of the mechanical stapler (gold cartridge) by the assistant for the division of the vessel.

The inferior and the superior borders of mediastinal branch of the pulmonary artery were dissected, and the artery was encircled with a vessel-loop and transected with stapler (gold cartridge). The connective tissue and station R10 nodes were harvested to obtain a proper exposure of the angle between the right main bronchus and RUL bronchus. After the identification of the inferior border of the bronchus, the fissure with the middle lobe was separated with staplers (purple cartridge). Finally, the bronchus was resected with a purple reload mechanical stapler. After completion of lobectomy, the specimen was extracted inside a plastic bag through the utility incision.

Postoperative course was uneventful. The patient was discharged on POD 2 after chest tube removal. Pathologic report showed no residual disease in the specimen (yp T0, ypN0).

Considering the optimal response to neoadjuvant therapy and the radical surgery, no adjuvant treatments were indicated in multidisciplinary discussion. The patient is currently alive without evidence of recurrence 8 months after surgery.

\section{Case 2}

\section{Case presentation}

A former smoker, 69-year-old male patient with previous history of surgically treated duodenal GIST, renal clear cell carcinoma, and COPD was found to have a $12 \mathrm{~mm}$ pulmonary nodule in the RUL with enlarged paratracheal, pretracheal and subcarinal lymph nodes at follow up chest CT scan. Head and abdomen CT scans were negative. A total-body 18-FDG-PET scan showed pathologic uptake only at the level of R4 nodes (SUVmax 15), which were biopsied through cervical mediastinoscopy, and resulted positive for metastasis from pulmonary adenocarcinoma.

The clinical staging was cTx/1a, cN2; the local multidisciplinary lung cancer board addressed the patient to neoadjuvant treatment with 2 cycles of carboplatin and vinorelbine. The following restaging with chest $\mathrm{CT}$ and PET scans showed an excellent response to the treatment (SUVmax 8.5 at R4 nodes). The patient was scheduled for robotic mediastinal and hilar lymphadenectomy with wedge resection of lung nodule, and possible lobectomy in case of primary lung cancer diagnosis at frozen-section. The routes of preoperative investigations and anesthesia were reported 


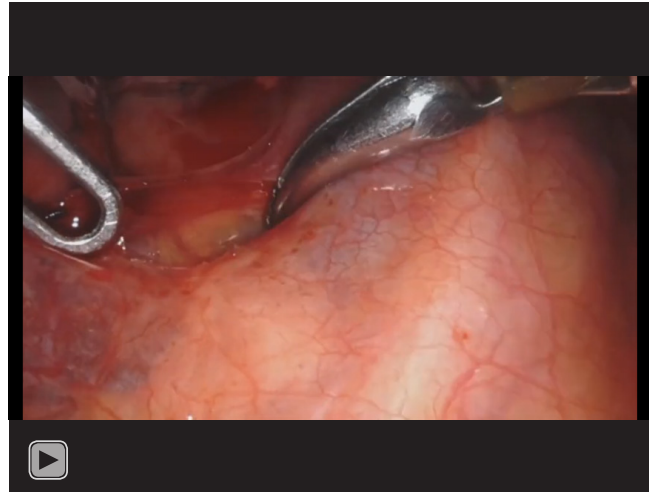

Video 2 Case 2, robotic-assisted superior vena cava tangential resection in a patient with mediastinal lymph node metastases from occult primary lung cancer.

in the appropriate section of Case 1.

\section{Surgical technique (Video 2)}

Surgery was carried out with four-arm robotic ParkVeronesi approach, as previously described. First, wedge resection of the RUL nodule and station R10 nodes were sent for frozen-section, both resulting negative for malignancy. Hence, the RUL was spared, and indication to radical mediastinal lymph node dissection was confirmed.

The mediastinal pleura was incised above the azygos vein, lateral to the superior vena cava (SVC), and paratracheal fatty tissue and station $\mathrm{R} 4$ nodes were dissected. Azygos vein was encircled and divided with Endo GIA stapler to provide a better exposure of the Barety lodge. After removal of metastatic R4 and R2 lymph nodes, unexpected subcentimetric invasion of the lateral wall of the SVC was encountered. Lymphadenectomy was completed, with persistence of a small residual area of pathologic tissue invading the SVC. The SVC was isolated distally and proximally to the infiltration. To achieve radicality, a tangential resection of the infiltrated SVC wall was performed with mechanical stapler (vascular reload) introduced by the bed assistant through the posterior robotic port (Figure 4). Surgery was completed with dissection of station 7 nodes.

No complications occurred during the postoperative course. The patient was discharged on the second POD. Histological examination confirmed lung adenocarcinoma metastases at R4 and R2 paratracheal stations as well as in resected SVC wall (ypTx, ypN2). Subsequently the patient underwent adjuvant radiotherapy cycles on the

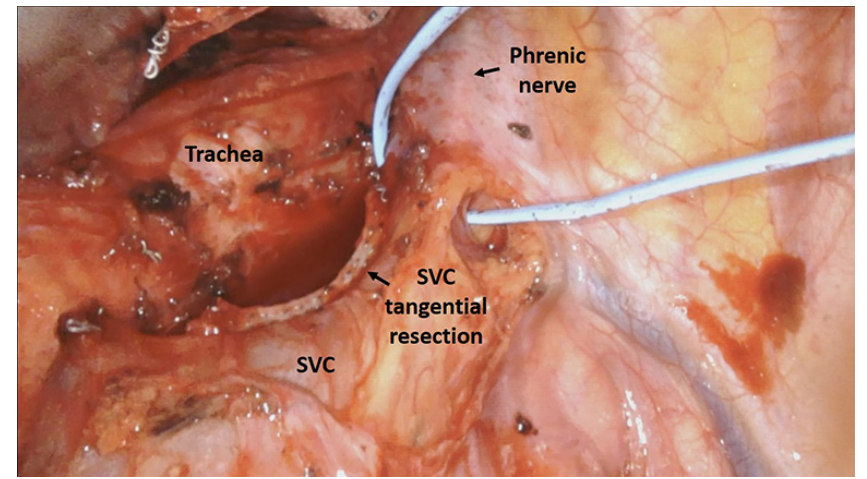

Figure 4 Case 2, tangential resection of SVC lateral side invaded by metastatic R2 and R4 lymph nodes. SVC, superior vena cava.

mediastinum. Two years after surgery, the patient is alive and free of disease.

\section{Discussion}

According to the IASLC definition released in 2005 (40), a lung resection can be considered complete when adequate free resection margin and systematic nodal dissection are obtained. Gagliasso et al. (41) validated this classification and demonstrated that patients with incomplete resection have a 3 -fold risk of mortality compared to those radically resected. Incomplete resection resulted an independent predictor of poor prognosis in a trial investigating the role of surgery in stage IIIB NSCLC (42).

From the analysis of the existing literature, there are well-recognized advantages of robotic surgery over VATS approach when facing with locally advanced NSCLC. First, RATS allows a better nodal dissection and thus more accurate staging and completeness of resection. As shown in Case 1, optimal visualization and better handeye coordination provided by $3 \mathrm{D}$-view and fine-movements of robotic arms were determinant factors in achieving complete dissection of hilar and mediastinal stations 'enbloc' with surrounding fatty tissue.

Secondly, most Authors agree that robotic surgery has indisputable benefits in tissue dissection, in particular in patients who received induction treatments. Despite the presence of bulky lesions, neoplastic neoangiogenesis, fibrosis induced by neoadjuvant therapy or secondary to diagnostic mediastinoscopy, or infiltration of major vascular structures, the rate of conversion is generally contained. In the past, SVC invasion in patients with N2-disease 
was considered a contraindication to surgery because of the poor survival rate and the technical challenges when carried out in non-experienced Centers (43). Nonetheless, it was demonstrated that long-term survival is achievable in patients undergoing induction therapy followed by partial SVC resection or prosthetic implantation, with acceptable rates of perioperative complications (44). $\mathrm{Xu}$ and colleagues (45) reported that tangential SVC resection with mechanic stapler is feasible during VATS surgery, but multiple N2-involvement might represent a contraindication for a minimally invasive approach. According to our experience, this problem may be solved proceeding with a robotic approach. In Case 2 , the curved bipolar dissector allowed fine dissection of fibrotic planes resulting from induction therapy and previous mediastinoscopy, leaving behind a small portion of tumor infiltrating the wall of SVC. Again, the possibility of rapid conversion of the utility incision to an open thoracotomy gave us enough safety to control potential major bleeding.

Finally, most papers comparing robotic surgery with open thoracotomy show significant reduction in postoperative length of stay. The reduced surgical trauma translates into faster recovery even in these patients who normally suffer a general impairment due to induction therapy. This is a key factor to enhance the possibility to undergo adjuvant therapy, even if evidence of increased long-term survival is still lacking $(21,23)$.

Several robotic approaches for lung resection have been described in the literature so far. According to the American Association of Thoracic Surgeons (AATS) Guideline Committee consensus statement (46), the distinguishing features of each technique are the eventual use of a utility incision, and the number of robotic arms employed during the operation.

Dylewski et al. and Cerfolio et al. $(17,47)$ described a complete portal approach for pulmonary lobectomy in 2011. While the former use 3 robotic arms (CPRL-3), an additional fourth arm (CPRL-4) was judged helpful from the latter to improve the retraction of pulmonary parenchyma. Moreover, robotic ports are placed along a single intercostal space to reduce postoperative pain. According to the Author opinion, carbon dioxide provides exposure of hilar structures, facilitates tissue dissection, and increases the internal working space. In both CPRL techniques, the approach to the hilum follows a posterioranterior route. At the end of the operation, the surgical specimen is removed from an additional trans- or supradiaphragmatic incision.
The RATS approach was first described in 2006 by Park and colleagues (48), and later modified by our group (16). Four robotic arms are introduced through 3 ports and a 3 -cm long utility incision at the fourth intercostal space (RAL-4). The dissection of hilar structures is carried out from the anterior aspect with a fissureless technique for upper lobes (39). Through the utility incision, the bed assistant can introduce accessory thoracoscopic instruments and remove the specimen without rib spreading. Moreover, a rapid conversion to open approach is possible in case of intraoperative major vascular accidents. $\mathrm{CO}_{2}$ is not routinely used; however, a rubber cover for the soft tissue retractor allows insufflation in selected cases when needed.

Regardless of the type of approach, the steep learning curve and financial aspects are known limitations of robotic surgery. Therefore, a solid expertise in the management of limited disease is of primary importance for a successful adoption of robotic surgery as a therapeutic option of locally advanced NSCLC.

\section{Conclusions}

Robotic surgery emerged as an appropriate approach to locally advanced NSCLC. In experienced centers, the technical characteristics of the system (3D-vision, widerange movements, and fine dissection) allow safe control even when facing bulky and infiltrating lesions in patients who underwent neoadjuvant therapy. Although the results of retrospective series are promising, multicentric prospective randomized trials are required.

\section{Acknowledgments}

The authors thank Dr. Francesca Rossetti for language review.

Funding: None.

\section{Footnote}

Provenance and Peer Review: This article was commissioned by the Guest Editor (Federico Rea) for the series "Alternative Surgical Approaches for Challenging Cases in Thoracic Surgery" published in fournal of Visualized Surgery. The article has undergone external peer review.

Conflicts of Interest: All authors have completed the ICMJE uniform disclosure form (available at https:// jovs.amegroups.com/article/view/10.21037/jovs-20-114/ 
coif). The series "Alternative Surgical Approaches for Challenging Cases in Thoracic Surgery" was commissioned by the editorial office without any funding or sponsorship. GV serves as an unpaid editorial board member of fournal of Visualized Surgery from June 2018 to May 2020. And she reports grants from INAIL, during the conduct of the study; personal fees from Medtronic, Ab Medica, and Johnson \& Johnson; grants from Intuitive, outside the submitted work. The authors have no other conflicts of interest to declare.

Ethical Statement: The authors are accountable for all aspects of the work in ensuring that questions related to the accuracy or integrity of any part of the work are appropriately investigated and resolved. All procedures performed in this study were in accordance with the Helsinki Declaration (as revised in 2013). The manuscript is waived from patient informed consent according to the ethics committee or institutional review board.

Open Access Statement: This is an Open Access article distributed in accordance with the Creative Commons Attribution-NonCommercial-NoDerivs 4.0 International License (CC BY-NC-ND 4.0), which permits the noncommercial replication and distribution of the article with the strict proviso that no changes or edits are made and the original work is properly cited (including links to both the formal publication through the relevant DOI and the license). See: https://creativecommons.org/licenses/by-nc-nd/4.0/.

\section{References}

1. Morgensztern D, Ng SH, Gao F, et al. Trends in stage distribution for patients with non-small cell lung cancer: a National Cancer Database survey. J Thorac Oncol 2010;5:29-33.

2. Lung, pleural and thymic tumours. In: Brierley JD, Gospodarowicz MK, Wittekind C. editors. UICC TNM classification of malignant tumours, 8th ed. OxfordHoboken: Wiley, 2017:106-12.

3. Huber RM, De Ruysscher D, Hoffmann H, et al. Interdisciplinary multimodality management of stage III nonsmall cell lung cancer. Eur Respir Rev 2019;28:190024.

4. National Comprehensive Cancer Network. Non-Small Cell Lung Cancer (Version 3.2020). [Accessed 27th March 2020]. Available online: https://www.nccn.org/ professionals/physician_gls/pdf/nscl.pdf

5. Postmus PE, Kerr KM, Oudkerk M, et al. Early and locally advanced non-small-cell lung cancer (NSCLC): ESMO
Clinical Practice Guidelines for diagnosis, treatment and follow-up. Ann Oncol 2017;28:iv1-iv21.

6. Heineman DJ, Daniels JM, Schreurs WH. Clinical staging of NSCLC: current evidence and implications for adjuvant chemotherapy. Ther Adv Med Oncol 2017;9:599-609.

7. Maniwa T, Shintani Y, Okami J, et al. Upfront surgery in patients with clinical skip N2 lung cancer based on results of modern radiological examinations. J Thorac Dis 2018;10:6828-37.

8. Bryan DS, Donington JS. The Role of Surgery in Management of Locally Advanced Non-Small Cell Lung Cancer. Curr Treat Options Oncol 2019;20:27.

9. Bott MJ, Yang SC, Park BJ, et al. Initial results of pulmonary resection after neoadjuvant nivolumab in patients with resectable non-small cell lung cancer. J Thorac Cardiovasc Surg 2019;158:269-76.

10. Albain KS, Swann RS, Rusch VW, et al. Radiotherapy plus chemotherapy with or without surgical resection for stage III non-small-cell lung cancer: a phase III randomised controlled trial. Lancet 2009;374:379-86.

11. Marulli G, Verderi E, Zuin A, et al. Outcomes and prognostic factors of non-small-cell lung cancer with lymph node involvement treated with induction treatment and surgical resection. Interact Cardiovasc Thorac Surg 2014;19:256-62.

12. Yang CF, Adil SM, Anderson KL, et al. Impact of patient selection and treatment strategies on outcomes after lobectomy for biopsy-proven stage IIIA pN2 non-small cell lung cancer. Eur J Cardiothorac Surg 2016;49:1607-13.

13. Nwogu CE, D'Cunha J, Pang H, et al. VATS lobectomy has better perioperative outcomes than open lobectomy: CALGB 31001, an ancillary analysis of CALGB 140202 (Alliance). Ann Thorac Surg 2015;99:399-405.

14. Menna C, De Falco E, Teodonio L, et al. Surgical wound-site inflammation: video-assisted thoracic surgery versus thoracotomy. Interact Cardiovasc Thorac Surg 2019;28:240-6.

15. Li S, Zhang W, Yang Z, et al. Systemic Inflammation Score as a Novel Prognostic Indicator for Patients Undergoing Video-Assisted Thoracoscopic Surgery Lobectomy for Non-Small-Cell Lung Cancer. J Invest Surg 2021;34:428-40.

16. Veronesi G, Galetta D, Maisonneuve P, et al. Four-arm robotic lobectomy for the treatment of early-stage lung cancer. J Thorac Cardiovasc Surg 2010;140:19-25.

17. Cerfolio RJ, Bryant AS, Skylizard L, et al. Initial consecutive experience of completely portal robotic pulmonary resection with 4 arms. J Thorac Cardiovasc 
Surg 2011;142:740-6.

18. Kent $M$, Wang $T$, Whyte $R$, et al. Open, video-assisted thoracic surgery, and robotic lobectomy: review of a national database. Ann Thorac Surg 2014;97:236-42; discussion 242-4.

19. Yang HX, Woo KM, Sima CS, et al. Long-term Survival Based on the Surgical Approach to Lobectomy For Clinical Stage I Nonsmall Cell Lung Cancer: Comparison of Robotic, Video-assisted Thoracic Surgery, and Thoracotomy Lobectomy. Ann Surg 2017;265:431-7.

20. Veronesi G. Robotic thoracic surgery: technical considerations and learning curve for pulmonary resection. Thorac Surg Clin 2014;24:135-41.

21. Park BJ, Yang HX, Woo KM, et al. Minimally invasive (robotic assisted thoracic surgery and video-assisted thoracic surgery) lobectomy for the treatment of locally advanced non-small cell lung cancer. J Thorac Dis 2016;8:S406-13.

22. Glover J, Velez-Cubian FO, Toosi K, et al. Perioperative outcomes and lymph node assessment after induction therapy in patients with clinical N1 or N2 non-small cell lung cancer. J Thorac Dis 2016;8:2165-74.

23. Veronesi G, Park B, Cerfolio R, et al. Robotic resection of Stage III lung cancer: an international retrospective study. Eur J Cardiothorac Surg 2018;54:912-9.

24. Li C, Hu Y, Huang J, et al. Comparison of robotic-assisted lobectomy with video-assisted thoracic surgery for stage IIB-IIIA non-small cell lung cancer. Transl Lung Cancer Res 2019;8:820-8.

25. Huang J, Li C, Li H, et al. Robot-assisted thoracoscopic surgery versus thoracotomy for $\mathrm{c}-\mathrm{N} 2$ stage NSCLC: short-term outcomes of a randomized trial. Transl Lung Cancer Res 2019;8:951-8.

26. Louie BE, Wilson JL, Kim S, et al. Comparison of VideoAssisted Thoracoscopic Surgery and Robotic Approaches for Clinical Stage I and Stage II Non-Small Cell Lung Cancer Using The Society of Thoracic Surgeons Database. Ann Thorac Surg 2016;102:917-24.

27. Mariolo AV, Casiraghi M, Galetta D, et al. Robotic Hybrid Approach for an Anterior Pancoast Tumor in a Severely Obese Patient. Ann Thorac Surg 2018;106:e115-6.

28. Qiu T, Zhao Y, Xuan Y, et al. Robotic sleeve lobectomy for centrally located non-small cell lung cancer: A propensity score-weighted comparison with thoracoscopic and open surgery. J Thorac Cardiovasc Surg 2020;160:838-46.e2.

29. Coşgun T, Kaba E, Ayalp K, et al. An antiquated contraindication for minimally invasive lung surgery: No place to staple the bronchus. Turk Gogus Kalp Damar
Cerrahisi Derg 2019;27:521-5.

30. Geraci TC, Ferrari-Light D, Wang S, et al. Robotic Sleeve Resection of the Airway: Outcomes and Technical Conduct using Video Vignettes. Ann Thorac Surg 2020;110:236-40.

31. Qiu T, Zhao Y, Xuan Y, et al. Robotic-assisted doublesleeve lobectomy. J Thorac Dis 2017;9:E21-5.

32. Pan X, Gu C, Yang J, et al. Robotic double-sleeve resection of lung cancer: technical aspects. Eur J Cardiothorac Surg 2018;54:183-4.

33. Marulli G, Faccioli E, Mammana M, et al. Predictors of nodal upstaging in patients with cT1-3N0 non-small cell lung cancer (NSCLC): results from the Italian VATS Group Registry. Surg Today 2020;50:711-8.

34. Asamura H, Chansky K, Crowley J, et al. The International Association for the Study of Lung Cancer Lung Cancer Staging Project: Proposals for the Revision of the $\mathrm{N}$ Descriptors in the Forthcoming 8th Edition of the TNM Classification for Lung Cancer. J Thorac Oncol 2015;10:1675-84.

35. Yazgan S, Ucvet A, Gursoy S, et al. Single-station skip-N2 disease: good prognosis in resected non-small-cell lung cancer (long-term results in skip-N2 disease). Interact Cardiovasc Thorac Surg 2019;28:247-52.

36. Kim AW, Liptay MJ, Bonomi P, et al. Neoadjuvant chemoradiation for clinically advanced non-small cell lung cancer: an analysis of 233 patients. Ann Thorac Surg 2011;92:233-41; discussion 241-3.

37. Melek H, Çetinkaya G, Özer E, et al. Pathological complete response after neoadjuvant/induction treatment: where is its place in the lung cancer staging system?. Eur J Cardiothorac Surg 2019;56:604-11.

38. Brunelli A, Kim AW, Berger KI, et al. Physiologic evaluation of the patient with lung cancer being considered for resectional surgery: Diagnosis and management of lung cancer, 3rd ed: American College of Chest Physicians evidence-based clinical practice guidelines. Chest 2013;143:e166S-e190S.

39. Veronesi G, Novellis P, Bottoni E, et al. Robotic Lobectomy: Right Upper Lobectomy. Oper Tech Thorac Cardiovasc Surg 2017;21:249-68.

40. Rami-Porta R, Wittekind C, Goldstraw P, et al. Complete resection in lung cancer surgery: proposed definition. Lung Cancer 2005;49:25-33.

41. Gagliasso M, Migliaretti G, Ardissone F. Assessing the prognostic impact of the International Association for the Study of Lung Cancer proposed definitions of complete, uncertain, and incomplete resection in non-small cell lung 
cancer surgery. Lung Cancer 2017;111:124-30.

42. Collaud S, Provost B, Besse B, et al. Should surgery be part of the multimodality treatment for stage IIIB nonsmall cell lung cancer? J Surg Oncol 2018;117:1570-4.

43. Spaggiari L, Tessitore A, Casiraghi M, et al. Survival after extended resection for mediastinal advanced lung cancer: lessons learned on 167 consecutive cases. Ann Thorac Surg 2013;95:1717-25.

44. Sato H, Soh J, Hotta K, et al. Is Surgery after Chemoradiotherapy Feasible in Lung Cancer Patients with Superior Vena Cava Invasion? Ann Thorac Cardiovasc Surg 2018;24:131-8.

45. Xu X, Qiu Y, Pan H, et al. Resection of the sidewall of

doi: 10.21037/jovs-20-114

Cite this article as: Muriana P, Perroni G, Novellis P, Veronesi G.

Robotic surgery for locally advanced non-small cell lung cancer.

J Vis Surg 2021;7:26. superior vena cava using video-assisted thoracic surgery mechanical suture technique. J Thorac Dis 2016;8:612-6.

46. Cerfolio R, Louie BE, Farivar AS, et al. Consensus statement on definitions and nomenclature for robotic thoracic surgery. J Thorac Cardiovasc Surg 2017;154:1065-69.

47. Dylewski MR, Ohaeto AC, Pereira JF. Pulmonary resection using a total endoscopic robotic video-assisted approach. Semin Thorac Cardiovasc Surg 2011;23:36-42.

48. Park BJ, Flores RM, Rusch VW. Robotic assistance for video-assisted thoracic surgical lobectomy: technique and initial results. J Thorac Cardiovasc Surg 2006;131:54-9. 